\title{
Enfoque tecnológico, cerámica y supervivencia de prácticas precolombinas: el ejemplo cañari (Ecuador)
}

Technologie céramique et persistance de pratiques précolombiennes

l'exemple cañari

Équateur

Ceramic technology and persistance of prehispanic practices: the Cañari

example (Ecuador)

\section{Catherine Lara}

\section{(2) OpenEdition}

Journals

Edición electrónica

URL: https://journals.openedition.org/bifea/11769

DOI: $10.4000 /$ bifea.11769

ISSN: 2076-5827

\section{Editor}

Institut Français d'Études Andines

Edición impresa

Fecha de publicación: 1 abril 2020

Paginación: 107-127

ISSN: 0303-7495

Referencia electrónica

Catherine Lara, «Enfoque tecnológico, cerámica y supervivencia de prácticas precolombinas: el ejemplo cañari (Ecuador)», Bulletin de l'Institut français d'études andines [En línea], 49 (1) | 2020, Publicado el 08 agosto 2020, consultado el 24 agosto 2021. URL: http://journals.openedition.org/ bifea/11769 ; DOl: https://doi.org/10.4000/bifea.11769

\section{(c) 9 (†) $\ominus$}

Les contenus du Bulletin de l'Institut français d'études andines sont mis à disposition selon les termes de la licence Creative Commons Attribution - Pas d'Utilisation Commerciale - Pas de Modification 4.0 International. 


\title{
Enfoque tecnológico, cerámica y supervivencia de prácticas precolombinas: el ejemplo cañari (Ecuador)
}

\author{
Catherine Lara*
}

\begin{abstract}
Resumen
La referencia a comunidades alfareras contemporáneas para interpretar contextos cerámicos prehispánicos se ha vuelto común en la arqueología andina. Esta práctica trae asimismo a colación los interrogantes de los fenómenos de continuidad o discontinuidad entre hábitos productivos precolombinos y actuales, así como de las implicaciones de estos fenómenos para la comprensión de la historia reciente de los grupos involucrados. La identificación e interpretación de persistencias o rupturas requiere asimismo una metodología adecuada. A través del caso cañari (Sierra Sur del Ecuador), se propone aquí uno de los enfoques de la antropología de las técnicas francófona como alternativa posible.
\end{abstract}

Palabras clave: etnoarqueología, enfoque tecnológico, tecnología cerámica, cañaris

\section{Technologie céramique et persistance de pratiques précolombiennes : l'exemple cañari (Équateur)}

\section{Résumé}

La référence aux communautés de potiers contemporaines pour interpréter des contextes céramiques préhispaniques s'est répandue au sein de l'archéologie andine. Cette pratique met en avant les questions des phénomènes de rupture ou de continuité entre les traditions productives précolombiennes et actuelles, ainsi que des implications de ces phénomènes pour la compréhension de l'histoire récente des groupes concernés. L'identification et l'interprétation de persistances ou de ruptures requiert néanmoins une méthodologie adaptée. À travers le cas d'étude des Cañaris (Sierra sud de l'Équateur), il est proposé ici que l'une des approches de l'anthropologie des techniques francophone est une alternative possible.

Mots-clés : ethnoarchéologie, approche technologique, technologie céramique, Cañaris

* Investigadora principal, Instituto Francés de Estudios Andinos - IFEA (UMIFRE 17 MEAE / CNRS USR 3337 América Latina). E-mail: catherine.lara@cnrs.fr 


\title{
Ceramic technology and persistance of prehispanic practices: the Cañari example (Ecuador)
}

\begin{abstract}
The reference to contemporary potters' communities as a means to interpret prehispanic ceramic contexts has become frequent in Andean archaeology. This practice raises the questions of the phenomenon of a break or continuity between precolumbian and modern production habits, as well as the topic of the implications of this phenomenon regarding the recent history of the groups under consideration. The identification and interpretation of persistence or break requires an appropriate methodology and I argue here that techné, one of the approaches popular in francophone anthropology, is a promising alternative.
\end{abstract}

Keywords: ethnoarchaeology, technological approach, ceramic technology, Cañaris

\section{TÉCNICAS PASADAS Y PRESENTES DE ELABORACIÓN CERÁMICA EN LA ARQUEOLOGÍA ANDINA}

Los primeros rastros del interés manifestado por arqueólogos hacia comunidades de alfareros andinas contemporáneas parecen encontrarse desde finales del siglo XIX (Ramón, 2008: 478). Desde aquel entonces, este tipo de estudios se desarrolló y multiplicó en el área andina. En su conjunto, estos aportes están encaminados a identificar la mayor variabilidad posible de factores que pueden influir en la organización de la producción cerámica de manera general, es decir, que permitirían potencialmente interpretar cualquier contexto arqueológico. Se destacan asimismo los estudios que buscan explorar el papel de los factores naturales en las prácticas alfareras (por ejemplo Arnold, 1975: 189; Cremonte, 1989-1990: 119; Druc, 2000: 171; Sillar, 2009: 112). El rol de las creencias y representaciones sociocognitivas (Calvo Trias \& García Rossselló, 2014: 8; Cremonte, 1989: 126) o de los factores históricos y sociopolíticos (Ramón \& Bell, 2013: 596; Sillar, 2009: 21) ocupa asimismo un puesto representativo en la literatura de la etnoarqueología cerámica regional.

La cultura material ligada a la producción cerámica es otro aspecto que ha captado significativamente el interés de las etnografías de alfareros realizadas por arqueólogos en los Andes. Lo ilustran, por ejemplo, los estudios de talleres actuales, orientados a abordar la organización de espacios de producción antiguos, y también a identificar el tipo de evidencia material que permitiría localizarlos en el registro arqueológico (Castellanos Montes, 2004; Vargas, 2018: 1). Se considera que las comunidades de ceramistas actuales —andinas o no-, constituyen referencias posibles para identificar y entender técnicas pasadas (Gabelmann, 2014: 44; Mohr Chávez, 1984: 162; Solórzano Venegas, 2015: 90; Villanueva Crisales, 2014: 22). Manuales de referencia como los de Balfet et al. (1989: 50), García Rosselló \& Calvo Trias (2013: 170) o Rice (2015: 148) integran ejemplos andinos en sus referentes de huellas técnicas. 
Más allá de posibles aplicaciones teórico-metodológicas generales, la alfarería andina actual plantea dos problemáticas ligadas a la historia y arqueología locales: 1) la existencia de fenómenos de continuidades o rupturas entre las prácticas alfareras actuales y aquellas heredadas de la época precolombina, y 2) las implicaciones de estos fenómenos en términos de identidad. Un sobrevuelo de la bibliografía sobre este tema evidencia posicionamientos distintos al respecto. Si bien escasos autores como Lanning (1963: 166) para Simbilá (costa norte del Perú) o Tschopik (1950: 217) en el caso de la cerámica aimara de Bolivia, afirman categóricamente la continuidad entre las técnicas antiguas y las actuales, la mayoría de los investigadores prefiere — quizás prudentemente- suponer esta continuidad: es el caso de García Rosselló (2007: 1938) con los mapuche en Chile, de Sjöman (1992: 23) en el Ecuador en general, de Bankes (1985: 269), de Shimada (1994: 298) o de Camino (in Sosa, 1984: 5) con los productores de cerámica paleteada de la costa norte del Perú.

Al parecer, la tendencia más generalizada en la arqueología andina para sugerir la existencia de continuidades entre técnicas pasadas y presentes en una localidad determinada se basa esencialmente en analogías entre herramientas arqueológicas y actuales (Druc, 2016: 3; Idrovo Urigüen, 2000: 61; Sabogal Wiesse, 1982: 36). La petrografía es usada como otro indicador potencial (Serrano, este volumen), al igual que la observación de fragmentos de cerámica arqueológica en comparación con material moderno (Druc, 2016: 13). Algunos autores como Christensen (1956: 187), Holm (1961: 185, 200) o Lanning (1963: 166) especifican los tipos de huellas que les permiten afirmar la presencia de continuidades. Otros como Reichel-Dolmatoff (1945: 430) en su estudio sobre los chamí de Colombia, son mucho más generales al respecto, conformándose con señalar la existencia de semejanzas entre la cerámica contemporánea y las vasijas arqueológicas.

Las posibles persistencias o rupturas entre prácticas técnicas pasadas y actuales conllevan implicaciones significativas en el marco de los estudios sobre los fenómenos de mezclas tecnológicas que pueden acompañar eventos de conquistas como la inca y la española (Sjöman, 1992: 238). Las prácticas artesanales son efectivamente reconocidas como vectores de expresión de identidad colectiva (Álvarez, 1987: 108) y/o individual, artística (Sosa, 1984: 25). Sosa (1984: 14) explica los casos de desaparición de técnicas alfareras prehispánicas en tanto que estas eran percibidas como una «amenaza» por los conquistadores. Otras formas cerámicas como las de Cuzco se equipararían, al contrario, a formas encubiertas de una «identidad andina 'denunciante'» (Sosa, 1984: 14). Para el altiplano cundiboyacense (Colombia), Therrien (1996: 95) sugiere que la subsistencia de prácticas alfareras precolombinas podría también explicarse por el alejamiento de las comunidades de artesanos en relación con los núcleos de poder coloniales.

De manera general, pese a la relevancia y actualidad de sus implicaciones, sobresale que la temática de las continuidades o rupturas entre técnicas alfareras precolombinas y modernas ha sido relativamente poco estudiada todavía (Shimada, 1994: 298; Sjöman, 1992: 23; Villanueva, 2014: 19). Argumentamos que el enfoque de la tecnología cerámica propuesto por Valentine Roux (2019), 
al que nos referiremos como «enfoque tecnológico» en las páginas siguientes, proporciona un marco teórico y metodológico completo para abordar esta temática1. A continuación, se explican las razones de esta afirmación, al detallar los fundamentos teórico-metodológicos de esta herramienta, antes de exponer su aplicación al caso de estudio cañari (Sierra sur del Ecuador).

\section{EL ENFOQUE TECNOLÓGICO, UNA ALTERNATIVA POSIBLE}

\section{1. Fundamentos}

El enfoque tecnológico se basa en el concepto de cadena operativa, o «camino técnico recorrido por un material desde su estado de materia prima hasta aquel de producto finito» (Cresswell, 1996: 43)². En el caso de la cerámica, este camino técnico cuenta con 6 acciones: extracción y preparación de la materia prima, manufactura (que comprende 2 etapas: esbozo ${ }^{3}$ y conformado4), acabado, tratamiento de superficie, decorado y quema (García Rosselló \& Calvo Trias, 2013: 52; Gosselain, 2000: 190)5.

El interés de las cadenas operativas radica en su potencial para evidenciar grupos sociales - definidos de manera amplia, es decir, a partir de criterios tan distintos como el género, la categoría étnica, social o la facción política, entre otros (Roux, 2007: 164)_. Así, para poder ejecutar cada una de las acciones de la cadena operativa, existe una gran variedad de técnicas y herramientas posibles. Por ejemplo, en el caso de la manufactura, se puede formar una vasija sobreponiendo varios rollos o cordeles de arcilla, con un molde, e inclusive combinando varias de estas técnicas que, tomadas de manera independiente, son universales. No obstante, a partir de cuantiosos casos etnográficos, el enfoque tecnológico ha observado que para cada acción de la cadena operativa, los alfareros de un mismo grupo social tienden a usar los mismos conjuntos de técnicas, formando combinaciones idiosincráticas (Gallay, 2011: 326). En otras palabras, cada grupo

1 Existen diferencias y, en ocasiones, desacuerdos acerca de diversos puntos tanto teóricos como metodológicos de las distintas propuestas existentes en torno al enfoque tecnológico aplicado a la cerámica. No abordaremos este tema aquí, por lo que nos concentramos únicamente en la propuesta de Roux (2019).

2 Varios elementos de los fundamentos de este enfoque fueron evidenciados previamente por distintos investigadores en diversas partes del mundo desde, al menos, el inicio del siglo XX. No nos extenderemos sobre este punto; señalamos nada más que el aporte del enfoque tecnológico consiste en haber sistematizado y enriquecido estas ideas previas en un marco teórico-metodológico completo, encaminado a identificar e interpretar tradiciones arqueológicas.

3 Volumen de arcilla hueco desprovisto de las características geométricas finales del recipiente, generalmente obtenido mediante operaciones destinadas a adelgazar las paredes (Courty \& Roux, 1995: 20).

4 Recipiente manufacturado que presenta las características geométricas finales de un recipiente, pero cuya superficie aún no ha sido sometida a acabado alguno (Courty \& Roux, 1995: 20).

5 Entendiéndose por «técnica» toda modalidad física de transformación de la materia prima (Roux, 2007: 158). 
social (en su calidad de comunidad de práctica) posee, a menudo, sus propias cadenas operativas características que las distinguen de los demás grupos y constituyen su tradición o «firma» característica.

¿Cómo explicar esta equivalencia sumamente frecuente entre grupos sociales y cadenas operativas? Esta regularidad se debería al proceso de aprendizaje y transmisión propio a todo quehacer técnico. Las observaciones etnográficas previamente citadas han recalcado que, en la gran mayoría de situaciones, el aprendizaje se realiza a través de un tutor, quien pertenece al mismo grupo social que el aprendiz (Bril, 2015: 111; Roux, 2007: 165). A través de mecanismos cognitivos, el aprendiz asimila los gestos indicados por el tutor. Al cabo de este aprendizaje, es muy difícil para el aprendiz considerar la puesta en práctica de otros tipos de gestos, en especial para la manufactura (para las demás acciones, que pueden ser aprendidas en un tiempo menor, el aprendiz podrá cambiar de técnica con mayor facilidad [Gallay, 2011: 326; Gosselain, 2000: 193]). Este proceso se repite de una generación a otra, lo cual contribuye a perpetuar las prácticas del grupo, convirtiéndolas en tradiciones.

Siendo así, la persistencia de una cadena operativa en torno a las prácticas de manufactura indica la perpetuación de una tradición asegurada dentro del mismo grupo mediante los procesos de transmisión y aprendizaje. Las variaciones que puedan surgir a lo largo del tiempo en torno a las formas y diseños o a los tratamientos de superficie, por ejemplo, reflejan a su vez los diversos fenómenos de contacto experimentados por un grupo (Coutet, 2014: 7; Huysecom \& Mayor, 1993: 312). A la inversa, los casos en los que se observa el remplazo repentino de una cadena operativa por otra totalmente distinta sugieren un fenómeno de cambio demográfico (Roux, 2015: 80). Por lo que el estudio diacrónico de las cadenas operativas de la producción cerámica de un lugar permite potencialmente identificar fenómenos de ruptura o continuidad, y sentar las bases de una reflexión sobre su significado.

\section{2. Componente etnográfico}

Como se vio, el enfoque tecnológico conlleva un componente etnográfico significativo, lo cual constituye un argumento adicional de su pertinencia dentro de problemáticas diacrónicas. No obstante, el uso de datos etnográficos en arqueología ha generado debate. A nuestro modo de ver, el enfoque tecnológico proporciona respuestas pertinentes a tres críticas principales.

A nivel teórico, se ha advertido justificadamente sobre el riesgo de acudir a analogías directas. Estas consisten en enfocarse en un fenómeno social actual, caracterizarlo a través de los indicadores materiales que lo acompañan en determinado contexto, $y$, al identificar estos mismos indicadores en contextos arqueológicos, deducir que estos últimos reflejan el mencionado factor social. La polisemia de los indicadores materiales en términos de factores sociales es, de hecho, un fenómeno bien conocido (Perlès, 2016: 224; Testart, 2012: 193). Para evitar este riesgo de analogía directa, el enfoque tecnológico optó por acudir a 
una instancia intermedia. Esta consiste en centrarse en un fenómeno transcultural (aprendizaje, transmisión, por ejemplo), el cual se va a buscar documentar con la mayor diversidad posible de casos etnográficos, con el propósito de identificar recurrencias o regularidades potencialmente útiles para entender contextos arqueológicos (Gallay, 2011: 210). La asociación sumamente frecuente entre cadena operativa y grupo social es una de ellas. La multiplicación de estudios en diversos contextos culturales permite también limitar, de alguna forma, los sesgos posibles acarreados por un trabajo llevado a cabo en un contexto cultural específico.

Por su parte, las tendencias de corte relativista plantean que es imposible plantear analogías entre sociedades distintas en el tiempo y el espacio, pues cada una de ellas es única e incomparable (Gosselain, 2016: 220). Esta perspectiva es, desde luego, incompatible con el enfoque tecnológico: si bien este reconoce que efectivamente hay particularidades individuales, sociales, históricas, la compleja simbiosis biológica y cultural propia del ser humano implica también la existencia de fenómenos compartidos por la totalidad de la especie, independientemente de la época o de la ubicación geográfica.

Desde una perspectiva deontológica, se ha criticado el trabajo con sociedades llamadas «tradicionales», en tanto que aquello significaría asumir implícitamente que se trata de grupos que no evolucionaron (Gosselain, 2016: 219; Lane, 2015: 22). En la medida en que el enfoque tecnológico se interesa en las modalidades cognitivas del aprendizaje y la transmisión, incluye estudios realizados en contextos occidentales de aprendizaje (adquisición de aptitudes deportivas, manejo de instrumentos musicales — ver Bril, 2015: 109_, sociología de redes —Manzo et al., 2018: 1116-).

\section{3. Protocolo metodológico}

¿Cómo hacer desde el enfoque tecnológico para comparar técnicas alfareras actuales con técnicas precolombinas? A continuación se resume el protocolo correspondiente al análisis del material antiguo, y luego, aquel de las técnicas actuales.

\section{3. 1. Cerámica arqueológica}

Para identificar cadenas operativas a partir de cerámica arqueológica, el análisis se hace idealmente en 3 etapas: clasificación de la muestra en grupos técnicos, grupos petrográficos y grupos morfo-estilísticos (Roux, 2019; 2017).

La clasificación en grupos técnicos busca identificar las técnicas de manufactura, acabado, tratamiento de superficie y quema empleadas para fabricar los recipientes originales de la muestra estudiada. Cada una de estas técnicas deja conjuntos específicos de huellas macro y microscópicas en la cerámica, cuya identificación permite, a su vez, aquella de las técnicas empleadas por los artesanos antiguos para cada acción de la cadena operativa. Estas huellas son conocidas gracias a referentes 
experimentales o etnográficos como aquellos mencionados en los manuales antes citados, a los que se puede agregar Rye (1981), Shepard (1956), y para estudios europeos más recientes y menos conocidos en la arqueología andina, Gelbert (2003: 75), Jeffra (2011: 115), Livingstone Smith (2007: 113), Martineau \& Pétrequin (2000: 337), Roux (2019: 158). Las huellas macroscópicas son perceptibles a simple vista o con la ayuda de dispositivos de observación de bajo aumento (Desbat \& Schmitt, 2011: 323). Puede tratarse, por ejemplo, de la topografía y color de la superficie de las vasijas o tiestos, así como de su modo de fractura (Roux, 2010: 8). Las trazas microscópicas, en cambio, solo pueden ser detectadas con la ayuda de una lupa binocular y/o una Dynolite. Se refieren a la microtopografía (tipo de granularidad y de estriación) y a la porosidad o vacíos visibles en los perfiles de los fragmentos (Desbat \& Schmitt, 2011: 326). Cabe subrayar que el enfoque tecnológico no hace diferencia alguna entre material diagnóstico y no-diagnóstico; todo material es diagnóstico, en la medida en que contiene huellas de fabricación macro y/o microscópicas potencialmente reveladoras.

La clasificación por grupos petrográficos se opera a partir de los grupos técnicos identificados previamente. Esta clasificación comienza por una observación preliminar de la pasta bajo la lupa binocular o la Dynolite, encaminada a describir las inclusiones (color, contornos, tamaño, densidad, distribución en la pasta) y las porosidades (forma, tamaño, densidad, distribución). De ser posible, estas observaciones son profundizadas mediante la toma de láminas delgadas, cuyo estudio permite, además, proporcionar indicaciones sobre la proveniencia de las fuentes de arcilla (Desbat \& Schmitt, 2011: 263).

Finalmente, dentro de cada grupo tecno-petrográfico identificado, se describen las formas y los diseños observados (Cauliez [2011: 56] o Mayor [1994: 184] proponen protocolos posibles, entre otros). Esta clasificación morfo-estilística permite comprobar si la variabilidad de grupos técnicos o tecno-petrográficos identificada se debe a factores culturales o funcionales (Roux, 2019: 226).

\section{3. 2. Cerámica actual}

Para poder comparar las técnicas identificadas en el material arqueológico con aquellas usadas por alfareros actuales, es preciso llevar a cabo un registro acucioso de cada acción del proceso de fabricación moderno: preparación de la pasta, manufactura, acabado, tratamientos de superficie, decorado y quema. La grilla descriptiva propuesta por Roux (2019: 41 —traducción al español: Lara, 2017: 65-) es una base descriptiva posible. Cada acción de la cadena operativa describe el método, las técnicas usadas (se toma en cuenta en especial el estado higrométrico de la pasta), los gestos, el procedimiento y las herramientas utilizadas. Aquello es posible mediante la observación directa del alfarero en el campo, complementada por un extenso registro fílmico y fotográfico. Un cuestionario basado en la grilla descriptiva permite asegurarse de haber integrado la mayor cantidad de parámetros posibles, incluyendo un registro comprehensivo de las prácticas productivas y de los contextos de aprendizaje. Es asimismo recomendable entrevistar al mayor número posible de alfareros, observar la fabricación de varios tipos de formas y 
para cada tipo de forma, idealmente, visualizar el mismo proceso varias veces. Se procede luego a recuperar muestras de las vasijas, para crear un referente experimental analizado en laboratorio, con el objetivo de registrar las huellas de cada vasija y asociarlas a sus técnicas de fabricación respectivas observadas en el campo.

Como se vio, la idea de comparar técnicas antiguas y actuales en torno a la problemática de la persistencia de prácticas precolombinas no es de ninguna manera novedosa en la arqueología andina. La tecnología cerámica presenta, no obstante, algunas especificidades a la hora de abordar este tipo de comparación. La relevancia otorgada a las analogías entre herramientas actuales y pasadas de una misma localidad es una de ellas. En efecto, una misma herramienta puede haber tenido usos distintos de una época a otra, o haber sido usada por grupos socioculturales diferentes. Por lo que este tipo de evidencia requiere pruebas adicionales para deducir la existencia de una continuidad técnica con un mayor rango de probabilidad. El enfoque tecnológico opta por concentrarse justamente en las huellas arqueométricas relacionadas con las técnicas de fabricación, visibles en las paredes de los recipientes. Esta perspectiva se asemeja, de hecho, a la propuesta de Roddick (2014: 286), encaminada a comparar huellas de fabricación antiguas y modernas en ceramios originarios de la cuenca sur del Titicaca (Bolivia). Desde luego, en todos los casos es preferible complementar la evidencia tecnológica proporcionada por la cerámica, respaldándose en diversos tipos de datos (otros componentes de la cultura material; de ser posible, datos etnohistóricos o lingüísticos etc.).

Valga recalcar que una misma huella puede haber sido ocasionada por técnicas distintas. No obstante, cada técnica se reconoce a través de un conjunto de huellas macro y microscópicas que le es propio. El reconocimiento de estos conjuntos requiere cierta capacitación y un entrenamiento continuo del ojo a partir de la observación de materiales modernos y arqueológicos. Asimismo, argumentar la puesta en práctica de determinada técnica a partir de material arqueológico exige proporcionar el listado de huellas que permite sustentar el argumento en cuestión. Una última particularidad del enfoque tecnológico radica en el papel otorgado a la petrografía, la cual es considerada como parte del análisis tecnológico.

\section{UNA APLICACIÓN ANDINA DE LA TECNOLOGÍA CERÁMICA: EL CASO DE ESTUDIO CAÑARI}

Los cañaris son un grupo étnico de aproximadamente 150000 personas actualmente asentado en la Sierra Sur del Ecuador (Quindi-Pichisaca, 2011: 33). Se identifican como los descendientes del grupo homónimo que ocupó este espacio en épocas precolombinas. En la ausencia de estudios genéticos diacrónicos comparativos, esta reivindicación es cuestionada por hipótesis principalmente basadas en datos etnohistóricos, las cuales argumentan que los cañaris precolombinos fueron prácticamente diezmados luego de las conquistas sucesivas protagonizadas por los incas y los españoles entre los siglos XV y XVI. Recordaremos primeramente 
los fundamentos de esta problemática, antes de explicar cómo se la trató desde el enfoque tecnológico y resumir los resultados obtenidos en consecuencia.

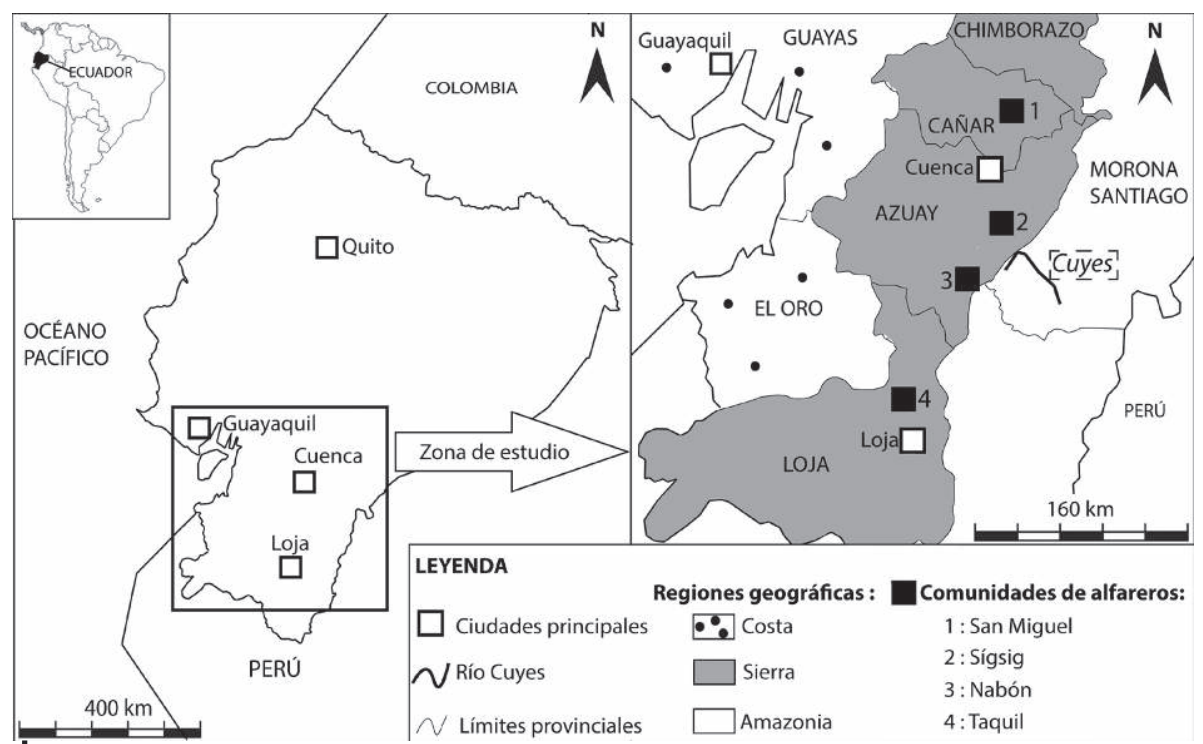

Figura 1 - Contexto geográfico de la zona de estudio (adaptado de Lara, 2018: 67)

(c) Catherine Lara

\section{1. De los protocañaris a los cañaris actuales}

Los cañaris precolombinos se asentaron principalmente en cuatro grandes valles interandinos ubicados en el sur del Ecuador, en las provincias actuales de Cañar y Azuay, y también en el sur de Chimborazo, el este de El Oro y Guayas, el oeste de Morona Santiago y el norte de Loja (fig. 1). Los conocimientos arqueológicos ligados a la génesis y desarrollo de la tradición cañari provienen esencialmente de la clasificación de los tipos cerámicos de la Sierra Sur del Ecuador iniciada a comienzos del siglo XX (Idrovo Urigüen, 2000: 53; Meyers, 1998: 172). Se asocia la aparición de los denominados «proto cañaris» a aquella del tipo cerámico llamado «Tacalshapa» (fig. 2), cuyos primeros rastros son reportados hacia el primer siglo antes de nuestra era (Idrovo Urigüen, 2000: 54).

Hacia el año 1000 de nuestra era, la llegada a esta zona de una ola migratoria proveniente de la Amazonia habría generado un desequilibrio social, y ocasionado la aparición de múltiples estilos cerámicos (Idrovo Urigüen, 2000: 61), que se desarrollaron paralelamente a Tacalshapa (ver también Lara, 2019: 194). Cashaloma (1000 d. C. hasta la conquista inca en el siglo XV), es el más emblemático de ellos (Fresco González, 1984: 185). A partir de esta ruptura del año 1000, se habla ya de «cañaris» propiamente (Idrovo Urigüen \& Gomis Santini, 2009: 40). En términos generales, protocañaris y cañaris son asociados a sociedades sedentarias y 


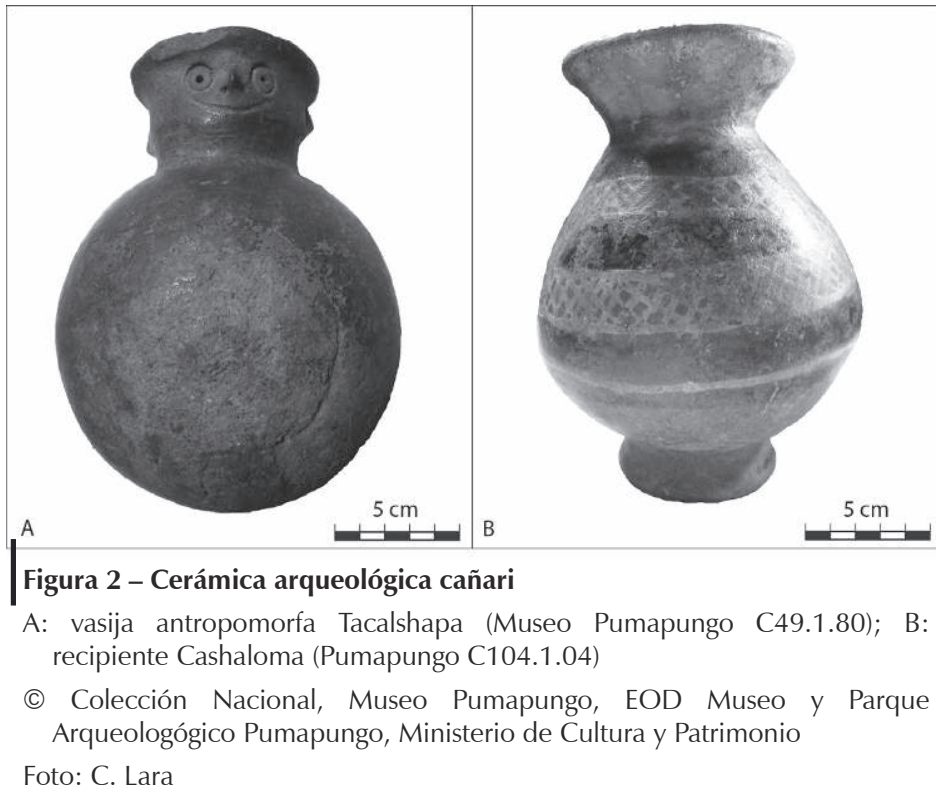

agrícolas de tipo cacical, con patrón de asentamiento disperso, especializadas más particularmente en la producción alfarera y metalúrgica. Estos grupos mantenían estrechos contactos con las sociedades vecinas costaneras, ubicadas entre el sur del Ecuador y Norte/norte del Perú actuales (Idrovo Urigüen, 2000: 56).

La documentación colonial proporciona información de primera mano sobre los cañaris «tardíos». Estos datos etnohistóricos presentan a los cañaris como un grupo etnolingüístico dividido en varias unidades políticas (Hirschkind, 2013: 43). A pesar de compartir tradiciones comunes (mito de origen por ejemplo Cieza de León, 1986 [1554]: 142—), cada una de estas unidades estaba asentada en su propio valle (u hoya), y encabezada por su propio dirigente (De Gauiria, 1965 [1582]: 282), lo cual habría contribuido a generar ciertas particularidades locales (Chacón Zaphán, 1990: 37) visibles en la cultura material (De Gallegos, 1965 [1582]: 278). Estas unidades habrían estado en contacto permanente entre ellas mediante intercambios (Hirschkind, 2013: 44) y conflictos (de Gallegos, 1965 [1582]: 275). Entre los documentos de esta época, se conoce una sola referencia a la existencia de comunidades de alfareros (de Gallegos, 1965 [1582]: 277), la cual no proporciona mayores informaciones sobre la identidad de sus artesanos o la técnica o tipos de vasijas fabricados. Entre 1463 y 1490, el territorio cañari es invadido por los incas, y, menos de un siglo más tarde (a partir de 1533), por los españoles (Hirschkind, 2013: 42, 59).

Existen dos hipótesis distintas acerca del devenir de los cañaris luego de estas dos conquistas.

La primera postula que los cañaris precolombinos fueron prácticamente erradicados por los incas y los españoles, y que los grupos que se autoproclaman actualmente cañaris provendrían más bien de los mestizajes sucesivos acontecidos entre 
grupos exógenos (Hirschkind, 2013: 59, ver también Burgos Guevara, 2003: 36). Este postulado se basa en estimaciones demográficas extraídas de documentos etnohistóricos, algunos de los cuales describen asimismo ampliamente los trastornos demográficos generados por estas conquistas. Los siguientes elementos de las tradiciones cañaris actuales demostrarían que estos no tendrían nada que ver con los cañaris precolombinos: son católicos, su vestimenta se compone de elementos derivados del traje español (Landívar, comunicación personal 2015, ver también Wachtel, 2013: 224), sus mitos y leyendas corresponden a la mitología inca, hablan español y quichua (un idioma derivado del quechua inca que remplazó a la lengua cañari, durante la presencia inca pero más que nada la evangelización hispana Hirschkind, 2013: 57, 58; Quindi Pichisaca, 2011: 36—).

La segunda hipótesis (Salomon, 2013: 37) reconoce efectivamente que muchos elementos de las tradiciones cañaris actuales se derivan de lo inca y lo español. Sin embargo, advierte también sobre los sesgos de las fuentes etnohistóricas en cuanto a lo que a datos demográficos se refiere. En algunos casos, por motivos políticos, éstos podían ser, de hecho, manipulados (por indígenas y por españoles —ver Poloni-Simard, 2006: 157-). Por otra parte, el uso frecuente de fuentes de segunda mano podía inducir a errores. Por lo que los recuentos que reflejan cataclismos demográficos deben ser manejados con precaución. Con base en testimonios coloniales provenientes de la zona cañari y en fuentes de la misma época encontradas en otros lugares del continente, se propone más bien que los cañaris tomaron el partido de adoptar voluntariamente elementos culturales incas y españoles, con el propósito de sobrevivir como colectivo frente a estas dos fuerzas dominantes.

\section{2. Aplicación del enfoque tecnológico y resultados}

Se optó por explorar la problemática de la persistencia o la desaparición de los cañaris precolombinos desde la perspectiva del enfoque tecnológico y la transmisión. Para ello, era preciso registrar, en primer lugar, las cadenas operativas de las cerámicas cañaris arqueológicas y, en segundo lugar, aquellas de la cerámica fabricada en la actualidad por los alfareros andinos del sur del Ecuador, para luego compararlas entre sí.

Dos tipos de conjuntos cerámicos arqueológicos fueron examinados. El primero corresponde a 117 objetos cañaris pertenecientes a las colecciones de 3 museos: en Francia, el museo del quai Branly-Jacques Chirac (fondo Paul Rivet); en el Ecuador, el museo Pumapungo de Cuenca y el museo municipal de Gualaquiza. Se conoce la procedencia de 101 de estos objetos. En su mayoría, fueron hallados en tumbas. El segundo conjunto corresponde a una muestra de 771 fragmentos provenientes de las excavaciones realizadas por quien suscribe en el valle del río Cuyes, en el extremo oriental del territorio cañari —sitios habitacionales y defensivos en su mayoría (Lara, 2017: 199)—.

Respecto al material actual, se escogieron las cuatro comunidades de alfareros más activas de la Sierra Sur del Ecuador (excluyendo los artesanos que usan el 
torno, cuyo origen se asocia a la colonización española —Lara, 2015: 33—). Dichas comunidades se distribuyen a lo largo de un segmento sur/norte de 160 kilómetros que abarca las comunidades de San Miguel de Porotos, Sígsig, Nabón y Taquil (fig. 1). Estas fueron localizadas luego de una prospección de dos semanas Ilevada a cabo por quien suscribe y una asistente en febrero de 2014. El trabajo de campo como tal fue realizado luego en dos temporadas: de marzo a junio de 2014, y de mayo a junio de 2015, es decir, durante más de seis meses. En total, 29 alfareros fueron visitados: 8 en San Miguel de Porotos (sobre 12 artesanos radicados en la comunidad), 4 en Sígsig (sobre 5), 1 en Nabón (sobre 4), y 16 en Taquil (sobre 67).

El cuadro 1 presenta una comparación sintética entre las cadenas operativas contemporáneas y precolombinas de los Andes del sur del Ecuador (para la descripción detallada de las técnicas modernas, de las huellas de fabricación registradas en el material actual y de aquellas identificadas en el material arqueológico, ver respectivamente Lara, 2017: 89; 131; 151 y Brazzero, este volumen para las técnicas actuales).

Siguiendo el orden de la cadena operativa, comenzando por la manufactura, sobresale que tanto en la cerámica prehispánica como en la actual, el modelado 6

Cuadro 1 - Comparación sintética de las cadenas operativas actuales y modernas de la Sierra Sur del Ecuador

\begin{tabular}{|c|c|c|c|}
\hline \multicolumn{2}{|c|}{ Acción cadena operativa } & Cerámica actual & Cerámica precolombina \\
\hline \multicolumn{2}{|c|}{ Preparación de la materia prima } & Arcilla + desgrasante & $\begin{array}{l}\text { Arcilla }+ \text { desgrasante? } \\
\text { (material excavado) }\end{array}$ \\
\hline \multirow[t]{2}{*}{ Manufactura } & ESBOZO & $\begin{array}{l}\text { Modelado + acordelado } \\
\text { Modelado }\end{array}$ & Modelado + acordelado \\
\hline & CONFORMADO & \multicolumn{2}{|c|}{ Golpeado } \\
\hline \multicolumn{2}{|l|}{ Acabado } & $\begin{array}{l}\text { Con golpeador } \\
\text { (cerámica) sobre pasta } \\
\text { en estado coriáceo } \\
\text { rehumedecido } \\
\end{array}$ & $\begin{array}{l}\text { Sobre pasta en estado } \\
\text { coriáceo (rehumedecido?) }\end{array}$ \\
\hline \multicolumn{2}{|c|}{ Tratamiento de superficie } & \multicolumn{2}{|c|}{ Ninguno, engobe, bruñido } \\
\hline \multicolumn{2}{|c|}{ Diseños } & \multicolumn{2}{|c|}{ En superficie, en hueco, de relieve } \\
\hline \multicolumn{2}{|l|}{ Formas } & $\begin{array}{l}\text { Ollas, cazuelas, jarras, } \\
\text { tortilleros, floreros, } \\
\text { cántaros, platos, sartenes, } \\
\text { tazas, jarras sin cuello, } \\
\text { tapas }\end{array}$ & $\begin{array}{l}\text { Ollas ( } \neq \text { tipos de cuellos), } \\
\text { cuencos, copas, vasos, } \\
\text { botellas }\end{array}$ \\
\hline \multicolumn{2}{|l|}{ Quema } & $\begin{array}{l}\text { A cielo abierto, } \\
\text { estructuras de quema } \\
\text { cerradas, hornos }\end{array}$ & $\begin{array}{l}\text { ¿A cielo abierto? (hipótesis } \\
\text { sugerida por el análisis } \\
\text { petrográfico del material } \\
\text { excavado) }\end{array}$ \\
\hline
\end{tabular}

(C) Catherine Lara

6 Pueden existir diferencias entre los distintos países hispanos en torno a los términos usados para nombrar las distintas técnicas, por lo que es mejor definirlas en caso de duda. Por modelado, se 
(fig. 3) interviene en el esbozo conjunto de la base y el cuerpo (salvo en Nabón actualmente para el cuerpo).

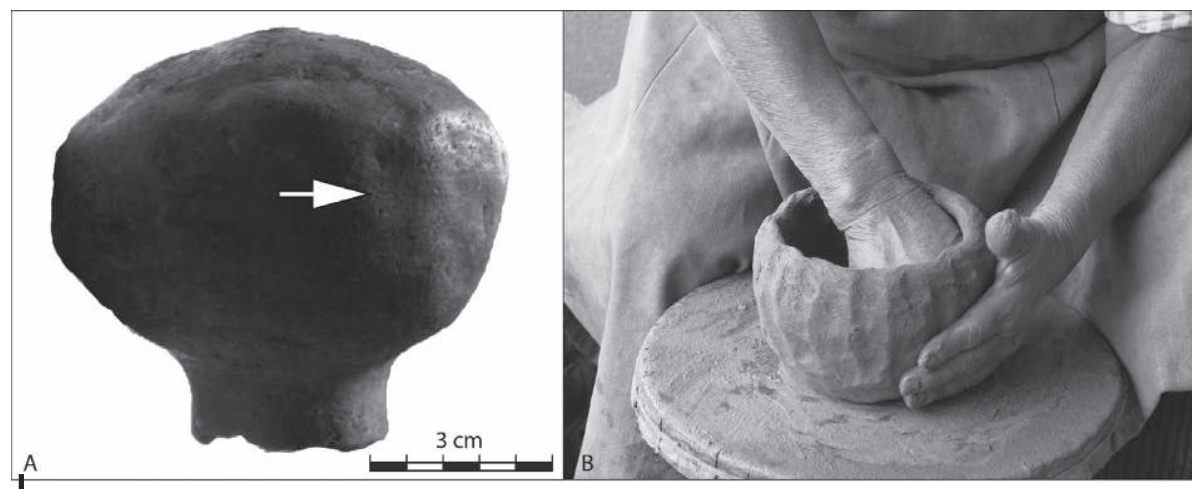

Figura 3 - Depresiones características de la técnica del modelado

A: recipiente cañari arqueológico (Pumapungo C679.1.80); B: recipiente actual (Taquil)

(C) foto 3A: Colección Nacional, Museo Pumapungo, EOD Museo y Parque Arqueologógico Pumapungo, Ministerio de Cultura y Patrimonio

Foto: C: Lara

La técnica del conformado por golpeado, en cambio, es empleada en el conjunto de la Sierra Sur del Ecuador, independientemente de la época (fig. 4).

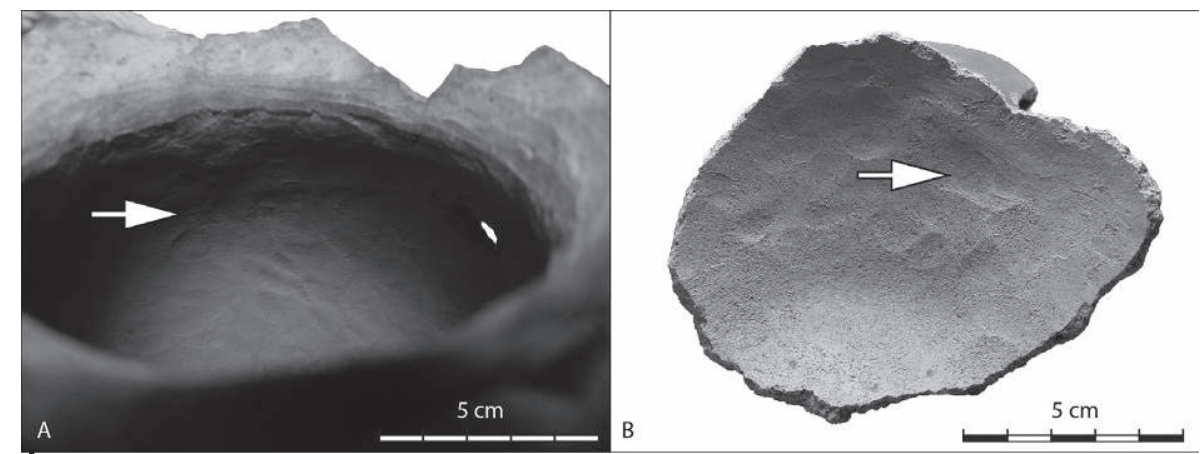

Figura 4 - Huellas características de la técnica del golpeado

A: recipiente cañari arqueológico (Pumapungo C25.1.80); B: recipiente actual (Sígsig)

(C) foto 4A: Colección Nacional, Museo Pumapungo, EOD Museo y Parque Arqueologógico Pumapungo, Ministerio de Cultura y Patrimonio. Foto: C: Lara.

(C) foto 4B: C. Lara

entiende aquí aquella técnica de esbozo que se ejerce sobre un volumen elemental conformado por una masa arcillosa homogénea, en el que — en este caso- se forma primeramente un hueco con el puño, antes de estirar y adelgazar lo que vendrán a ser las paredes. 
Se añadirá que los golpeadores encontrados en los contextos arqueológicos cañaris son los mismos que aquellos usados por los alfareros modernos (fig. 5). De la misma manera, el acordelado del cuello es omnipresente (fig. 6), salvo en Nabón hoy en día. El bruñido es recurrente, salvo en las localidades actuales de Nabón y Sígsig. Por su parte, los diseños presentan variaciones significativas en el sentido en que, en la época prehispánica, los diseños en superficie eran predominantes (hoy en día, solo se los encuentra en Nabón), mientras que actualmente, los motivos en hueco son los más comunes.

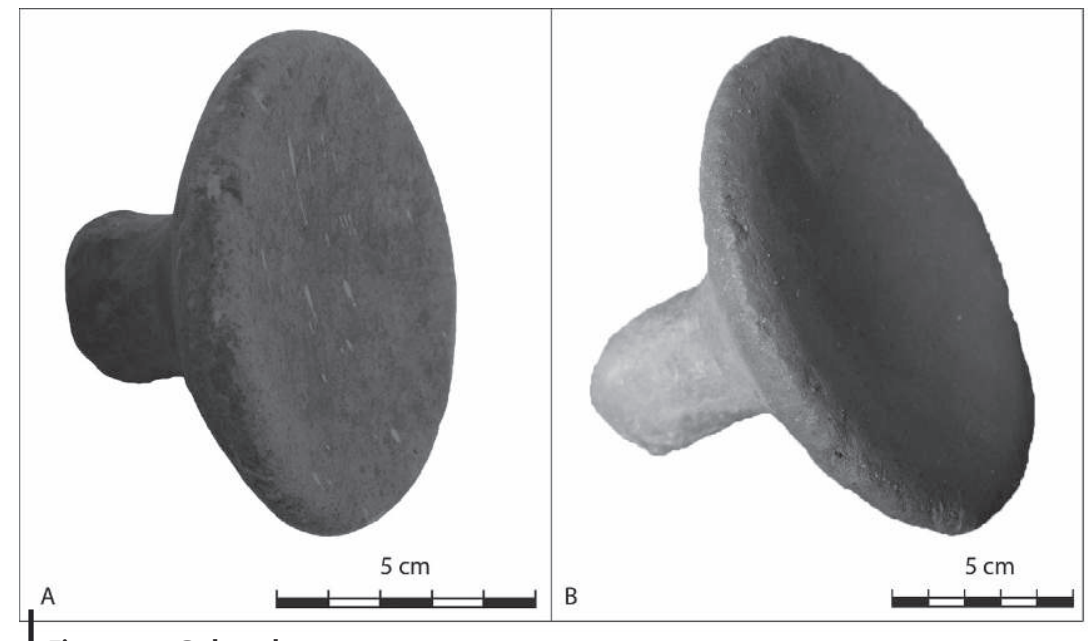

Figura 5 - Golpeador externo

A: arqueológico (Pumapungo C4777.1.80); B: moderno (Taquil)

(C) foto 5A: Colección Nacional, Museo Pumapungo, EOD Museo y Parque Arqueologógico Pumapungo, Ministerio de Cultura y Patrimonio

Foto: C. Lara

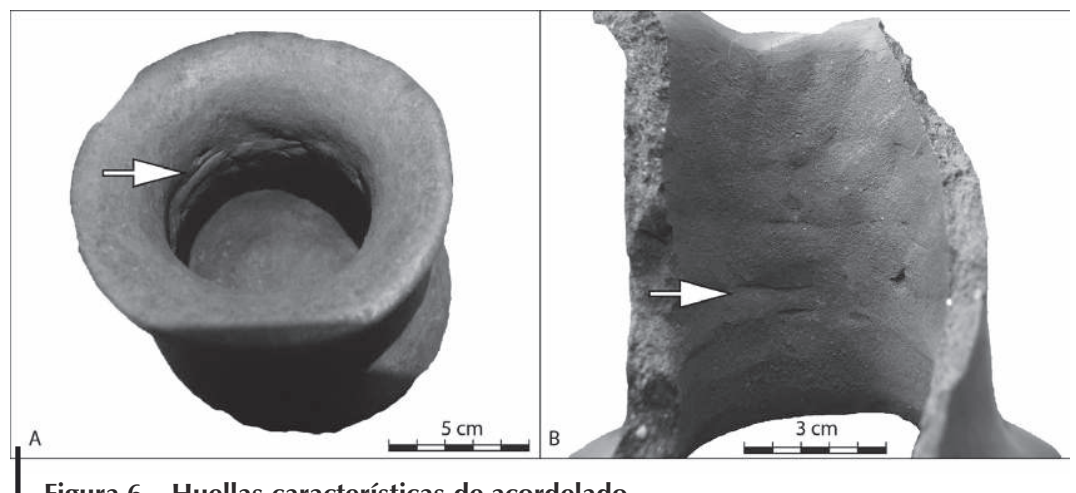

Figura 6 - Huellas características de acordelado

A: recipiente arqueológico (Pumapungo C782.1.80); B: recipiente actual (Taquil)

(C) foto 6A: Colección Nacional, Museo Pumapungo, EOD Museo y Parque Arqueologógico Pumapungo, Ministerio de Cultura y Patrimonio

Foto: C. Lara 
Respecto a las formas, el repertorio morfológico evidencia una variabilidad significativa diacrónica y sincrónicamente (fig. 7).

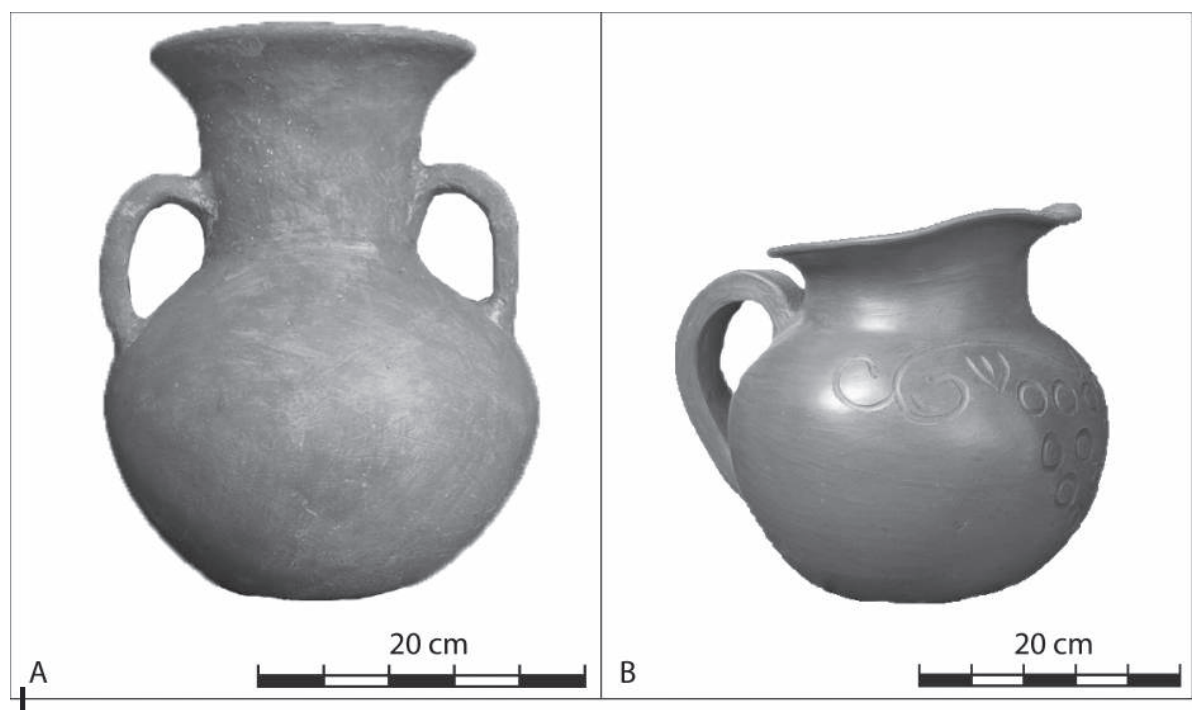

Figura 7 - Recipientes modernos de la Sierra sur del Ecuador

A: Sígsig; B: Taquil

(C) Catherine Lara

En resumen, la manufactura es la acción que más similitudes evidencia entre la alfarería actual y antigua, seguida por el acabado y los tratamientos de superficie. Por su parte, las formas y los diseños varían considerablemente en el tiempo y en el espacio. Desde los fundamentos del enfoque tecnológico (transmisión de tutor a aprendiz pertenecientes al mismo grupo social) y el fenómeno de estabilidad de las cadenas operativas en torno a la manufactura, esta observación sugiere que hubo efectivamente una transmisión entre alfareros cañaris precolombinos y actuales. Las diferencias observadas entre las cerámicas modernas y pasadas —en particular referente a las formas y los diseños $y$, en menor medida, a los tratamientos de superficie y el esbozo-, serían a su vez el reflejo de los fenómenos endógenos y exógenos vividos por los alfareros cañaris a lo largo de su historia (llegada de los incas, de los españoles, migraciones y contactos subsiguientes correspondientes al periodo republicano).

Desde luego, la alfarería es un aspecto puntual de la cultura y de la expresión de identidad, más aún en un caso complejo como el cañari. Un fenómeno similar al que describimos aquí para la cerámica ha sido resaltado por los trabajos lingüísticos de Howard en la Sierra sur del Ecuador, los cuales «demuestran la persistencia de un substrato cañari en el léxico quichua, con sus rasgos fonológicos asociados, a lo largo del siglo XX y por lo menos hasta comienzos del siglo XXI» (Howard, 2019: 199). Panorama que confirma el interés de multiplicar estudios 
parecidos en otros ámbitos de los saberes tradicionales, como la metalurgia o el trabajo textil, en donde se sospecha la supervivencia de prácticas precolombinas (Martínez Borrero, 1993: 162).

El hecho de que la cadena operativa precolombina ligada al golpeado se haya mantenido hasta hoy en la Sierra sur del Ecuador, sugiere que, al menos, algunos alfareros cañaris tienen que haber sobrevivido lo suficiente como para transmitir su conocimiento a una descendencia que, sin duda, pasó luego por un proceso de mestizaje biológico, pero que siguió perpetuando la tradición. Lo cual nos invitaría a matizar de alguna forma el escenario «radical» de una desaparición total de los cañaris —al menos culturalmente_-, y a seguir explorando más bien la hipótesis de una transformación identitaria progresiva, en la que algunas tradiciones se perdieron, y otras sobrevivieron, aunque con cambios (ver también Polony-Simard [2006: 558] para un análisis etnohistórico profundizado de las diversas estrategias sociales de adaptación identitaria de mestizos e indígenas en el corregimiento de Cuenca durante la Colonia).

\section{A MANERA DE CONCLUSIÓN}

El caso cañari expuesto en la presente investigación confirma que el enfoque tecnológico es una herramienta particularmente pertinente en el marco de problemáticas relacionadas con la identificación de fenómenos de continuidad y ruptura existentes desde la época precolombina hasta la actualidad. En primer lugar, porque se trata de un método cuyas herramientas teóricas y metodológicas se basan fuertemente en contextos y materiales etnográficos. En segundo lugar, porque la tecnología cerámica presta especial atención al fenómeno diacrónico de la transmisión. Contextualizados en el marco de otros tipos de información existentes (en el caso cañari, datos etnohistóricos o lingüísticos por ejemplo), los datos «en bruto» proporcionados por la comparación entre cerámica arqueológica y actual, de acuerdo al enfoque tecnológico, permiten sentar las bases de una reflexión en torno a los motivos posibles de los fenómenos de ruptura o persistencia eventualmente identificados. Esta mirada tecnológica sobre la historia de los colectivos, a través de su cultura material, ofrece perspectivas particularmente enriquecedoras para el entendimiento de las identidades andinas contemporáneas.

Se percibe en ocasiones cierto temor dentro de las ciencias sociales al hablar de la preservación de tradiciones ancestrales. En el tema de la continuidad de prácticas abordado aquí, la identificación de un fenómeno de persistencia en un ámbito determinado, de ninguna manera implica afirmar despectivamente que el grupo considerado no cambió, sino que permite más bien orientar la investigación hacia prácticas de adaptación frente a fenómenos como conquistas o mestizajes (ver Politis [2015: 68] sobre el potencial «dinamizante» del trabajo etnoarqueológico). En el caso cañari expuesto aquí, más allá de la simplificación inducida por escenarios como la simple continuidad entre pasado y presente o el etnocidio, se consolida la posibilidad de un grupo que también es actor de su historia (ver Solari, 2015: 61). 


\section{Agradecimientos}

A Valentine Roux (proyecto ANR DIFFCERAM ANR-12-CULT-0001-01) y Stéphen Rostain, directores de la tesis que originó este trabajo, realizada en la université Paris Nanterre (UMR 7055/ED395); a los alfareros de San Miguel, Sígsig, Nabón y Taquil; al Museo Pumapungo (Colección Nacional, EOD Museo y Parque Arqueológico Pumapungo, Ministerio de Cultura y Patrimonio) y al INPC.

\section{Referencias citadas}

ÁLVAREZ, S., 1987 - Artesanías y tradición étnica en la península de Santa Elena. Revista Artesanías de América, 25: 45-119.

ARNOLD, D., 1975 - Ceramic ecology of the Ayacucho basin, Peru: Implications for Prehistory. Current Anthropology, 16: 183-205.

BALFET, H., FAUVET-BERTHELOT, M.-F. \& MONZON, S., 1989 - Lexique et typologie des poteries pour la normalisation de la description des poteries, 146 pp.; París: Presses du CNRS.

BANKES, G. H. A., 1985 - The Manufacture and Circulation of Paddle and Anvil Pottery on the North Coast of Peru. World Archaeology, 17 (2): 269-277.

BRIL, B., 2015 - Learning to use tools: a functional approach to action. In: Francophone perspectives of learning through work. Conceptions, Traditions and Practices (L. Filliettaz \& S. Billett, eds.): 95-118; Heidelberg: Springer International Publishing.

BURGOS GUEVARA, H., 2003 - La identidad del pueblo cañari, de-construcción de una nación étnica, 91 pp.; Quito: Abya-Yala.

CALVO TRIAS, M. \& GARCÍA ROSSELLÓ, J., 2014 - Acción técnica, interacción social y práctica cotidiana: propuesta interpretativa de la tecnología. Trabajos de Prehistoria, 71 (1): 7-22.

CAMINO, L., 1984 - Introducción. In: El barro nos unió: arte y tecnología de la cerámica de Chulucanas, Piura (G. Sosa, ed.): 5-6; Piura: CIPCA.

CASTELLANOS MONTES, D., 2004 - Cultura material y organización espacial de la producción cerámica en Ráquira: un modelo etnoarqueológico, 95 pp.; Bogotá: Fundación de Investigaciones Arqueológicas Nacionales (FIAN)-Banco de la República.

CAULIEZ, J., 2011 - 2900-1900 av. n.-è. Une méthodologie et un référentiel pour un millénaire de produits céramiques dans le sud-est de la France, 126 pp.; Aix-enProvence: Éditions APPAM.

CHACÓN ZHAPÁN, J., 1990 - Historia del Corregimiento de Cuenca 1557-1777, 675 pp.; Cuenca: Banco Central del Ecuador.

CHRISTENSEN, R. T., 1956 - An archaeological study of the Illescas-Jubones coast of northern Peru and southern Ecuador, 416 pp. + 14 láminas; Arizona: Universidad de Arizona, Departamento de Antropología. Tesis de doctorado.

CIEZA DE LEÓN, P., 1986 [1554] - Crónica del Perú (primera parte), 352 pp.; Lima: Pontificia Universidad Católica del Perú, Academia Nacional de Historia.

COURTY, M.-A. \& ROUX, V., 1995 - Identification of Wheel Throwing on the basis of Ceramic Surface Features and Microfabrics. Journal of Archaeological Science, 22 (1): 17-50. 
COUTET, C., 2014 - La caractérisation techno-stylistique de la céramique de tradition Arauquinoïde en Guyane : une approche ethnoarchéologique de la céramique amérindienne. Karapa. Revue d'anthropologie des sociétés amérindiennnes anciennes, d'histoire et d'archéologie coloniale du bassin amazonnien et du plateau des guyanes, 3: 6-20.

CREMONTE, M.-B., 1989 - La alfarería tradicional actual: reflexiones y posibles aplicaciones para la Arqueología a través de dos casos de estudio. Runa, 19 (1): 117-133.

CRESSWELL, R., 1996 - Prométhée ou Pandore ? Propos de technologie culturelle, 393 pp.; París: Éditions Kimé.

DE GALLEGOS, G., 1965 [1582] - Relación que enbio a mandar su magestad se hiziese desta ciudad de Cuenca y de toda su provincia - San Francisco Pueleusi del Azogue. In: Relaciones geográficas de Indias, t. II - Perú (M. Jiménez de la Espada, ed.): $274-$ 278; Madrid: Ediciones Atlas.

DE GAUIRIA, M., 1965 [1582] - Relación que envió a mandar su majestad se hiciese de esta ciudad de Cuenca y de toda su provincia (Cañaribamba). In: Relaciones geográficas de Indias - Perú, t. II (M. Jiménez de la Espada, ed.): 281-287; Madrid: Ediciones Atlas.

DESBAT, A. \& SCHMITT, A., 2011 - Glossaire. In: La céramique, la poterie du Néolithique aux temps modernes (A. D'Anna, A. Desbat, D. Garcia, A. Schmitt \& F. Verhaeghe, eds.): 313-330; París: Éditions Errance.

DRUC, I., 2000 - ¿Shashal o no shashal? Esa es la cuestión. Etnoarqueología cerámica en la zona de Huari, Ancash. Bulletin de l'Institut Français d'Études Andines, 30 (1): 157-173.

DRUC, I., 2016 - Traditional potters from the Andes to Vietnam, 139 pp.; Blue Monnds, WL: Deep University Press.

FRESCO GONZÁLEZ, A., 1984 - La arqueología de Ingapirca (Ecuador): costumbres funerarias, cerámica y otros materiales, 203 pp.; Quito: Comisión del Castillo de Ingapirca, Consejo de Gobierno del Museo Arqueológico del Banco Central del Ecuador.

GABELMANN, O. U., 2014 - Diversas preferencias tecnológicas. Diversas preferencias tecnológicas. Reconstruyendo la cadena operatoria de la producción cerámica del período Formativo en Cochabamba, Bolivia. In: La rebelión de los objetos: enfoque cerámico (E. Espejo \& M. Eyzaguirre, eds.): 39-63; La Paz: Musef.

GALLAY, A., 2011 - Pour une ethnoarchéologie théorique : mérites et limites de l'analogie ethnographique, 389 pp.; París: Éditions Errance.

GARCÍA ROSSELLÓ, J., 2007 - La producción cerámica mapuche. Perspectiva histórica, arqueológica y etnográfica. In: Actas del $6^{\circ}$ Congreso Chileno de Antropología (Colegio de Antropólogos, ed.): 1932-1946; Valdivia: Colegio de Antropólogos.

GARCÍA ROSSELLÓ, J. \& CALVO TRIAS, M., 2013 - Making pots: el modelado de la cerámica y su potencial interpretativo, 477 pp.; Oxford: British Archaeological Reports.

GELBERT, A., 2003 - Traditions céramiques et emprunts techniques dans la vallée du fleuve Sénégal, 104 pp.; París: Maison des sciences de l'homme, Epistèmes.

GOSSELAIN, O., 2000 - Materializing identities: an African perspective. Journal of Archaeological Method and Theory, 7 (3): 187-217.

GOSSELAIN, O., 2016 - To hell with ethnoarchaeology! Archaeological Dialogues, 23 (2): 215-228.

HIRSCHKIND, L., 2013 - Historia de la población indígena del Cañar. Revista de Antropología, 20: 41-63. 
HOLM, O., 1961 - La técnica alfarera de Jatunpampa (Ecuador). Cuadernos de Historia y Arqueología, X (27): 153-210 (+ láminas).

HOWARD, R., 2019 - ¿Por qué nos roban nuestros fonemas? Sobrevivencia de la lengua cañari en el imaginario sociopolítico indígena. In: Léxico y contacto de lenguas en los Andes (L. Andrade Ciudad, Á. Ezcurra \& C. Garatea Grau, eds.): 185-202; Leck: Fondo editorial PUCP, Peter Lang.

HUYSECOM, É. \& MAYOR, A., 1993 - Les traditions céramiques du delta intérieur du Niger. In: Vallées du Niger (Société française de promotion artistique, ed.): 297-313; París: Réunion des Musées Nationaux.

IDROVO URIGÜEN, J., 2000 - Tomebamba, arqueología e historia de una ciudad imperial, 348 pp.; Quito: Banco Central del Ecuador-Dirección Cultural regional Cuenca.

IDROVO URIGÜEN, J. \& GOMIS SANTINI, D., 2009 - Historia de una región formada en el Austro del Ecuador y sus conexiones con el norte del Perú, 117 pp.; Cuenca: Prefectura del Azuay, Gobierno Provincial del Azuay.

JEFFRA, C., 2011 - The Archaeological Study of Innovation: An Experimental Approach to the Pottery Wheel in Bronze Age Crete and Cyprus, 329 pp.; Exeter: University of Exeter, Departamento de Arqueología. Tesis de doctorado.

LANE, P., 2015 - Peripheral vision: reflections on the death and rebirth of ethnoarchaeology. In: Breaking barriers. Proceedings of the $47^{\text {th }}$ Annual Chacmool Archaeological Conference (R. Crook, K. Edwards \& C. Hughes, eds.): 19-34; Calgary: the University of Calgary, Chacmool Archaeological Association of the University of Calgary.

LANNING, E. P., 1963 - A ceramic sequence for the Piura and Chira coast, North Peru. In: American Archaeology and Ethnology, 46: 135-278; Berkeley/Los Angeles: University of California Press.

LARA, C., 2015 - [Catálogo de la exposición «Presencia del pasado: la alfarería contemporánea del sureste del Ecuador»]. Yachac (número especial) 13: 47 pp.

LARA, C., 2017 - Aportes del enfoque tecnológico a la arqueología precolombina: pasado y presente de la alfarería en el valle del río Cuyes y su región (Andes sur-orientales del Ecuador), 240 pp.; Oxford: Archaeopress.

LARA, C., 2018 - Nouvelles perspectives sur les Cañaris d'hier et d'aujourd'hui : la céramique des Andes méridionales de l'Équateur de 100 av. J.-C. jusqu'à nos jours. Journal de la Société des américanistes, 104 (2): 65-104.

LARA, C., 2019 - Tacalshapa y Cashaloma: perspectivas del enfoque tecnológico. Revista de Historia, Patrimonio, Arqueología y Antropología de América, 1: 180-199.

LIVINGSTONE SMITH, A., 2007 - Chaîne opératoire de la poterie, références ethnographiques, analyses et reconstitution, X + 203 pp.; Tervuren: Musée royal de l'Afrique centrale.

MANZO, G., GABBRIELLINI, S., ROUX, V. \& NKIROTE M'MBOGORI, F., 2018 - Complex Contagions and the Diffusion of Innovations: Evidence from a Small-N Study. Journal of Archaeological Method and Theory, 25: 1109-1154.

MARTINEAU, R. \& PÉTREQUIN, P., 2000 - La cuisson des poteries néolithiques de Chalain (Jura), approche expérimentale et analyse archéologique. In: Arts du feu et productions artisanales (P. Pétrequin, P. Fluzin, J. Thiriot \& P. Benoit, eds.): 337-358; Antibes: APDCA.

MARTÍNEZ BORRERO, J., 1993 - La cultura popular en el Ecuador, tomo 1, Azuay, 236 pp.; Cuenca: CIDAP.

MAYOR, A., 1994 - Durées de vie des céramiques africaines : facteurs responsables et implications archéologiques. In: Terre cuite et société. La céramique, document technique, économique, culturel (D. Binder \& J. Courtin, ed.): 179-198; Juan-lesPins: APDCA. 
MEYERS, A., 1998 - La tradición Tacalshapa y la arqueología del Cañar, Sur del Ecuador. In: 50 Years Americanist Studies at the University of Bonn (S. Dedenbach-Salazar Sáenz, C. Arellano Hoffmann, E. König \& H. Prümers, eds.): 169-199; Bonn: Verlag Anton Saurwein.

MOHR CHÁVEZ, K. L., 1984 - Traditional pottery of Raqch'i, Cuzco, Peru: a preliminary study of its production, distribution and consumption. Ñawpa Pacha, Journal of Andean Archaeology, 22-23: 161-210.

PERLÈS, C., 2016 - La technologie lithique, de part et d'autre de l'Atlantique. Bulletin de la Société Préhistorique Française, 113 (2): 221-240.

POLITIS, G., 2015 - Reflections on contemporary ethnoarchaeology: reflexiones sobre etnoarqueología contemporánea. Pyrenae. Revista de prehistoria/Antiguitat de la la Mediterránea Occidental, 46 (1): 41-83.

POLONY-SIMARD, J., 2006 - El mosaico indígena. Movilidad, estratificación social y mestizaje en el Corregimiento de Cuenca (Ecuador) del siglo XVI al siglo XVIII, 605 pp.; Quito: Casa de Velásquez, Abya-Yala, IFEA.

QUINDI PICHISACA, A., 2011 - El territorio de la gran nación cañari. In: La nación cañari y sus expresiones culturales (J. Blankenship, ed.): 33-37; Estados-Unidos: NMAI/ Museo Nacional del Indígena Americano.

RAMÓN, G., 2008 - Producción alfarera en Piura (Perú): estilos técnicos y diacronía. Bulletin de I'Institut Français d'Études Andines, 37 (3): 477-509.

RAMÓN, G. \& BELL, M., 2013 - Re-placing plainware: Production and distribution of domestic pottery, and the narration of the pre-colonial past in the Peruvian Andes. Journal of Anthropological Archaeology, 32 (4): 595-613.

REICHEL-DOLMATOFF, G., 1945 - La manufactura de cerámica entre los Chamí. Boletín de Arqueología, 1 (1): 425-430.

RICE, P., 2015 - Pottery analysis, a sourcebook, 592 pp.; Chicago: The University of Chicago Press.

RODDICK, A., 2014 - Trazo e Itinerario: (Re) Definiendo la cadena operatoria de la producción alfarera en la cuenca sur del lago Titicaca. In: Rebelión de los objetos: enfoque cerámico. Anales de la reunión anual de Etnología XXVIII (E. Espejo \& M. Eyzaguirre, eds.): 279-302; La Paz: Musef.

ROUX, V., 2010 - Lecture anthropologique des assemblages céramiques, fondements et mise en oeuvre de l'analyse technologique. Les nouvelles de l'archéologie, 119: 4-9.

ROUX, V., 2015 - Cultural Transmission, Migration and Plain wheelmade Pottery in the Middle Bronze Age II Southern Levant. In: Plain Pottery Traditions of the Eastern Mediterranean and Near East: Production, Use, and Social Significance (C. Glatz, ed.): 69-90; Walnut Creek: Left Coast Press.

ROUX, V., 2017 - Not to throw the baby out with the bathwater. A response to Gosselain's article. Archaeological Dialogues, 24 (2): 225-229.

ROUX, V., 2019 - Ceramics and Society: a Technological Approach to Archaeological Assemblages, 329 pp.; Suiza: Springer.

RYE, O., 1981 - Pottery technology. Principles and reconstruction, 150 pp.; Washington: Taraxacum.

SABOGAL WIESSE, J. R., 1982 - La cerámica de Piura (t. 1), 263 pp.; Quito: Ladap.

SALOMON, F., 2013 - Ancestros, huaqueros y los posibles antecedentes del "Incaísmo" cañari. Revista de Antropología de la Casa de la Cultura del Azuay, 20: 6-40.

SHEPARD, A., 1956 - Ceramics for the Archaeologist, 414 pp.; Washington: Carnegie Institution of Washington. 
SHIMADA, I., 1994 - La producción de cerámica en Mórrope, Perú: productividad, especialización y espacio vistos como recursos. In: Tecnología y organización de la producción prehispánica en los Andes (I. Shimada, ed.): 295-319; Lima: Fondo Editorial de la Pontificia Universidad Católica del Perú.

SILLAR, B., 2009 - La saisonnalité des techniques. Saisonnalité et spécialisation artisanale dans les Andes. Techniques et culture. Revue semestrielle d'anthropologie des techniques, 52-53: 90-119.

SJÖMAN, L., 1992 - Vasijas de barro: la cerámica popular en el Ecuador, 406 pp.; Cuenca: CIDAP.

SOLARI PITA, M., 2015 - "Los caminos de la etnicidad: identidades de los descendientes de mitmas cañaris en la región andina (siglos XVI-XXI)". Antropología Andina Muhunchik - Jathasa, 2 (1): 44-71.

SOLÓRZANO VENEGAS, M. S., 2015 - El pasado en el presente. Métodos de elaboración cerámica vigentes en artefactos arqueológicos. Antropología cuadernos de investigación, 15: 81-91.

SOSA, G., 1984 - La cerámica. In: El barro nos unió: arte y tecnología de la cerámica de Chulucanas, Piura (L. Camino \& B. Revesz, eds.): 17-90; Piura: CIPCA.

TESTART, A., 2012 - Avant l'histoire. L'évolution des sociétés, de Lascaux à Carnac, 560 pp.; París: Gallimard.

THERRIEN, M., 1996 - Persistencia de prácticas indígenas durante la colonia en el altiplano cundiboyacense. Boletín del Museo del Oro, 40: 89-99.

TSCHOPIK, H., 1950 - An Andean Ceramic Tradition in Historical Perspective. American Antiquity, 15 (3): 196-218.

VARGAS, J. P., 2018, Ms. - Producción alfarera en el Austro ecuatoriano, una perspectiva etnoarqueológica. Ponencia presentada en la IX Reunión de Teoría Arqueológica de América del Sur (TAAS), Ibarra (Ecuador), 6 de junio del 2018.

VILLANUEVA CRIALES, J., 2014 - Moldeando la vida, la colección de cerámica del Museo Nacional de Etnografía y Folclore, según la cadena de producción, 496 pp.; La Paz: Museo Nacional de Etnografía y Folklore, Fundación Cultural del Banco Central de Bolivia.

WACHTEL, N., 2013 - La vision des vaincus. Les indiens du Pérou devant la conquête espagnole, 1530-1570, 395 pp.; París: Gallimard. 